\title{
Manejo interdisciplinario para una óptima rehabilitación oral.
}

\section{Interdisciplinary management for optimal oral rehabilitation.}

\author{
Mónica Badillo Barba,* Jorge Morales García,‡ Julio Magaña Quiñones,§ Jessica Berenice Flores Hernández,* \\ Gabriela Noemí Escutia Corona," María Fernanda Figueroa González"
}

\section{RESUMEN}

Las estrategias para el éxito en la rehabilitación bucal requieren de la interrelación de varias disciplinas que en conjunto logren resultados predecibles y duraderos. La visión individualizada de cada área de especialidad puede conllevar a no ofrecer la mejor alternativa de tratamiento, es por ello que la valoración, el diagnóstico y la planificación del caso clínico debe ser realizada por un equipo interdisciplinario para evitar esta situación y crear una sinergia en donde el «todo sea mayor que la suma de sus partes». El objetivo de este trabajo es presentar un caso clínico en el cual intervinieron varias áreas de especialidad: periodoncia, prostodoncia, cirugía oral y patología bucal, logrando devolver la función y la estética a través del manejo interdisciplinario.

Palabras clave: Cirugía preprotésica, prótesis inmediata, regularización de proceso alveolar, frenectomía, interdisciplinaria.

\section{ABSTRACT}

The strategies for success in oral rehabilitation require the interrelation of several disciplines, which together, achieve predictable and lasting results. The individualized view of each specialty area may lead to not offering the best treatment alternative, which is why the assessment, diagnosis, and planning of the clinical case must be carried out by an interdisciplinary team to avoid this situation and create a synergy in where the «whole is greater than the sum of its parts». The objective of this work is to present a clinical case where several areas of specialty intervened: periodontics, prosthodontics, oral surgery, and oral pathology, thus achieving the return of function and aesthetics through interdisciplinary management.

Keywords: Preprosthetic surgery, immediate prosthesis, alveolar process regularization, frenectomy, interdisciplinary.

\section{INTRODUCCIÓN}

$\mathrm{E}^{\prime}$ objetivo principal de la rehabilitación bucal es mejorar la función del sistema estomatognático en los pacientes que han tenido experiencias de disfunciones en la masticación, el habla o en la deglución y estética como consecuencia de la pérdida dentaria o por otras razones. El sistema masticatorio es un complejo sistema neuromuscular y esquelético que involucra la actividad de los músculos, nervios, huesos y los tejidos periodontales, ${ }^{1,2}$ por ello su reconstitución debe ser a partir de un trabajo interdisciplinario para lograr una rehabilitación que cumpla con los requerimientos estéticos, funcionales y biológicos.

La pérdida de dientes causa alteraciones tales como la disminución de la fuerza masticatoria, pérdida de la sensación propioceptiva, además de los desfavorables efectos estéticos en la zona facial, ${ }^{3}$ así como un impacto psicológico importante, ya que el paciente se ve privado

\footnotetext{
* Profesor investigador Departamento Atención a la Salud.

‡ Profesor investigador Departamento Atención a la Salud, Profesor invitado de la UNITEC y UIC.

$\S$ Profesor investigador Departamento Atención a la Salud, Profesor invitado de la UNITEC, ULA.

๑ Alumno.
}

Universidad Autónoma Metropolitana-Xochimilco.

Recibido: 08 de octubre de 2020. Aceptado: 25 de marzo de 2021.

Citar como: Badillo BM, Morales GJ, Magaña QJ, Flores HJB, Escutia CGN, Figueroa GMF. Manejo interdisciplinario para una óptima rehabilitación oral. Rev ADM. 2021; 78 (2): 106-114. https://dx.doi.org/10.35366/99287 

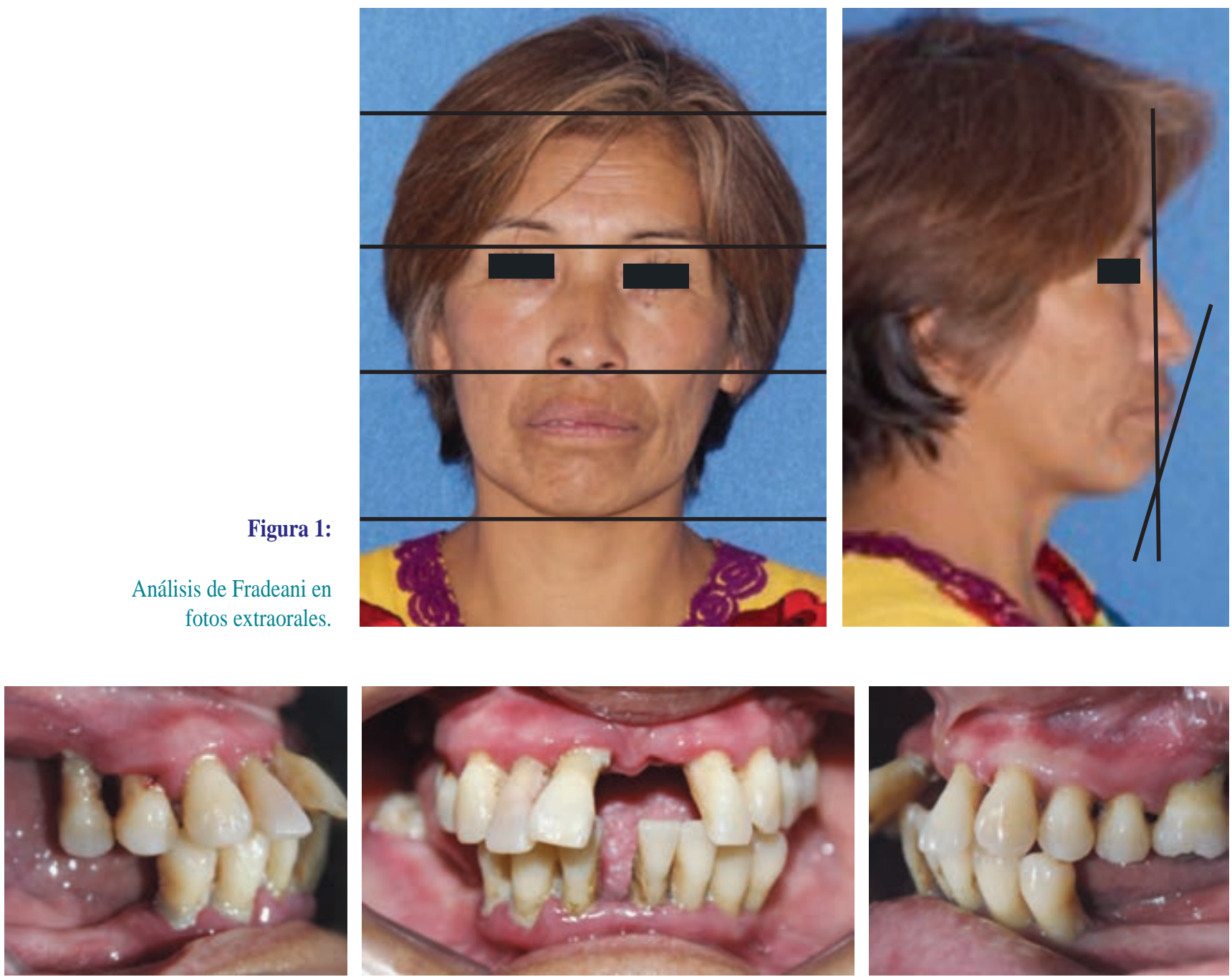

Figura 2: Fotos intraorales.

de una parte importante de su propia identidad y puede sufrir inadaptación, lo cual repercute en su bienestar psicosocial y, por ende, en la calidad de vida. Por lo tanto, la rehabilitación del paciente no sólo debe considerar un plan interdisciplinario de las áreas de especialidad estomatológica, sino que también es importante tener en cuenta el aspecto psicológico. ${ }^{4}$

La Organización Mundial de la Salud (OMS) menciona que la salud oral es fundamental para gozar de una buena salud general y una buena calidad de vida; se puede definir como la ausencia de dolor orofacial, cáncer de boca o de garganta, infecciones y llagas bucales, enfermedades periodontales, caries, pérdida de dientes y otras enfermedades y trastornos que limitan en la persona afectada la capacidad de morder, masticar, sonreír y hablar, al tiempo que repercuten en su bienestar psicosocial. ${ }^{5}$

La transición de dentición completa a condición desdentada es un proceso gradual, son varios los factores desencadenantes de la pérdida dental, como la edad, el estado sistémico, la enfermedad periodontal, los traumatismos, entre otros. ${ }^{6}$

La prótesis inmediata es una alternativa en la transición de la rehabilitación protésica definitiva; constituye una posibilidad para reducir la tragedia física y psíquica que se produciría en el desdentado, ya que permite la transformación del dentado natural al artificial, sin cambios faciales ni alteraciones estéticas. ${ }^{7}$ 
Entre las ventajas de esta prótesis inmediata se encuentran brindar un vendaje, proteger el coágulo, favorecer su epitelización, limitar la inflamación, la orientación del trabeculado óseo, así como la ubicación del diente en relación con el macizo craneofacial. El paciente se recupera del choque de las extracciones, al tiempo que se acostumbra al uso de la prótesis, a diferencia del procedimiento ordinario en el que el dolor y la incomodidad deben ser soportados en dos etapas diferentes. ${ }^{8}$

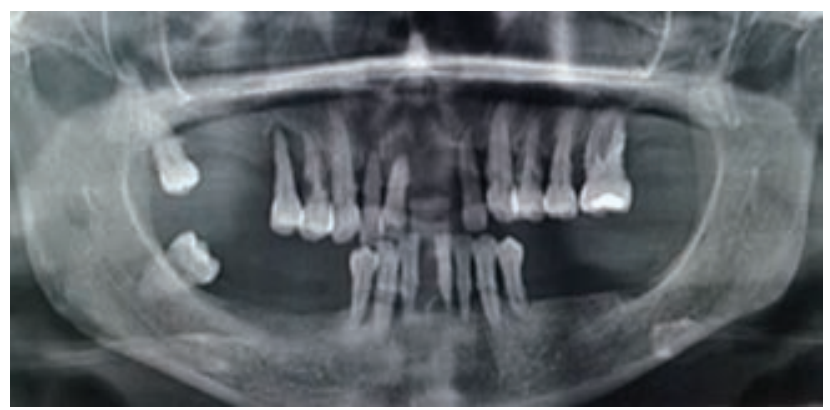

Figura 3: Ortopantomografía.
El tratamiento definitivo del desdentado total consiste básicamente en devolver las estructuras dentarias perdidas a través de medios artificiales como las prótesis totales, pese a que la terapia de los implantes ha provisto una solución para el edentulismo, las prótesis completas convencionales continúan siendo la mejor opción para la mayoría de los pacientes, en especial cuando existen limitaciones económicas. ${ }^{9}$

Las bases protésicas deberán ofrecer tres aspectos fundamentales: soporte, retención y estabilidad. La oclusión en la rehabilitación del desdentado total es fundamental, pues permitirá cumplir con la retención y estabilidad de las bases protésicas. Los distintos esquemas oclusales (balanceada, no balanceada, lineal o monoplano, lingualizada y mordida cruzada) han demostrado ser útiles dependiendo del caso a tratar; sin embargo, el esquema balanceado bilateral aún continúa siendo el principal esquema a utilizar en la mayoría de los casos de edentulismo total, ya que genera contactos dentales simultáneos durante los movimientos excéntricos y las fuerzas oclusales laterales generadas durante los movimientos son compartidas por todos los dientes y la articulación temporomandibular. ${ }^{10}$

Figura 4:

Placas base superior e inferior con rodillos.
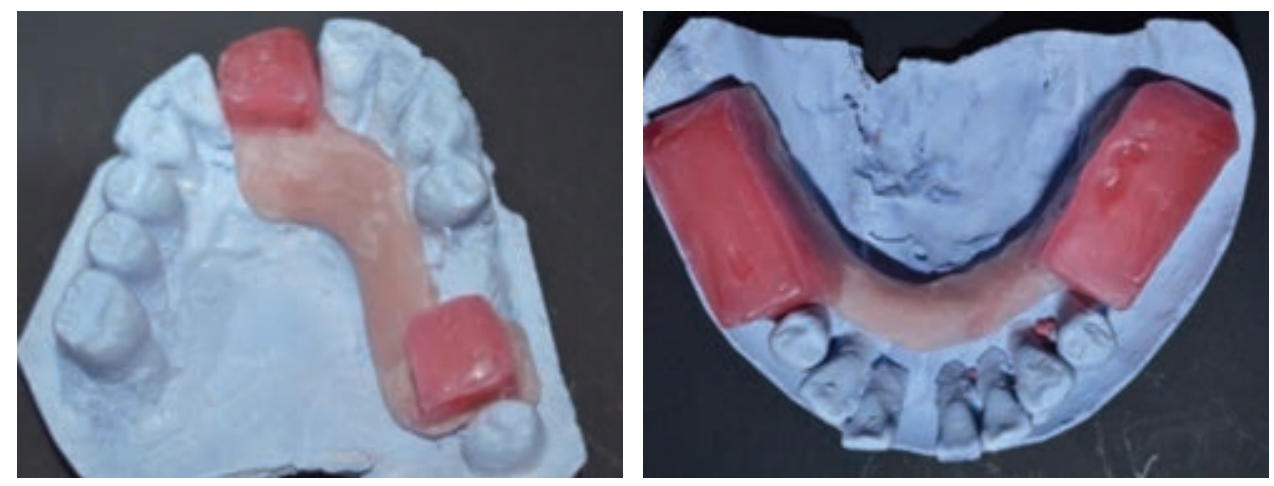

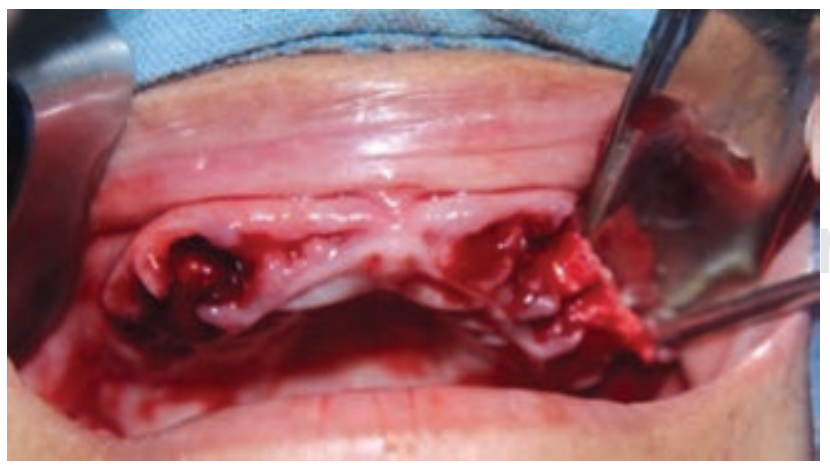

Figura 5: Cirugía preprotésica superior.

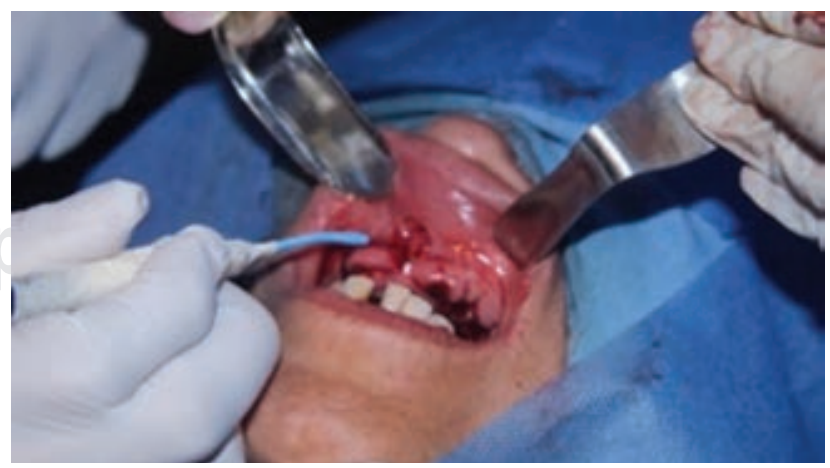

Figura 6: Frenectomía superior. 
El objetivo de este caso fue realizar una adecuada rehabilitación devolviendo la estética y la función a través del manejo interdisciplinario.

\section{PRESENTACIÓN DEL CASO}

Paciente femenino de 52 años que acude al Servicio Dental de la Clínica de San Lorenzo de la UAM-Xochimilco. El motivo de consulta fue «quiero arreglarme mis dientes», en el interrogatorio no refirió ninguna implicación sistémica, por lo que fue catalogada como paciente ASA1. En el análisis facial, de acuerdo con Fradeani, ${ }^{11}$ presenta tercios desproporcionados y protrusión labial (Figura 1).

A la exploración clínica intrabucal presenta cálculo dental supra- y subgingival, movilidad dental gene-

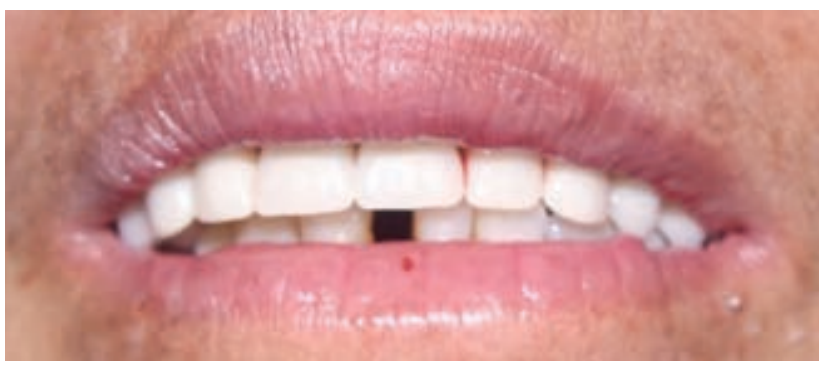

Figura 7: Colocación de prótesis inmediata superior. ralizada y frenillo bucal superior con inserción baja (Figura 2).

En el análisis radiográfico se observa una pérdida ósea entre 60 y 90\% casi de forma generalizada, el diente 18 y 48 completamente mesializado (Figura 3).

El plan de tratamiento consistió en cuatro fases:

1. Evaluación periodontal y análisis de modelos.

2. Cirugía preprotésica (extracciones múltiples, regularización de procesos alveolares y frenectomía) superior y colocación de prótesis inmediata.

3. Cirugía preprotésica (extracciones múltiples, regularización de procesos alveolares y frenectomía) inferior y colocación de prótesis inmediata.

4. Elaboración y colocación de prótesis definitivas.

\section{Evaluación periodontal y análisis de modelos}

El diagnóstico periodontal dado para la paciente fue:

Periodontitis crónica severa generalizada y de periodontitis estadio 4 grado A dentro de la nueva clasificación para las enfermedades periodontales de $2018 .{ }^{12}$

Se ferulizaron los dientes superiores con arco de acero $16 \times 24$ y resina fluida para tomar la impresión y realizar los modelos de estudio, en los cuales se elaborará la placa base con rodillos para establecer la dimensión vertical
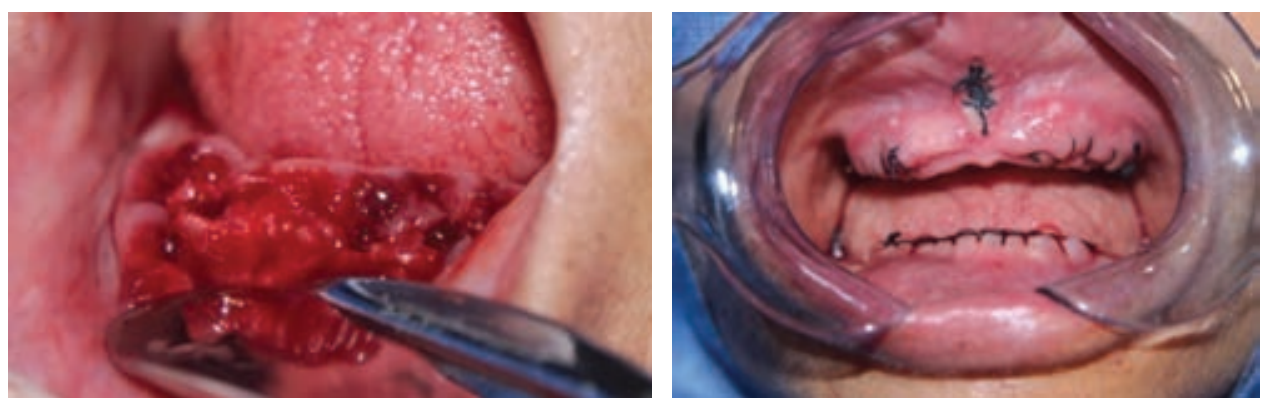

Figura 8:

Cirugía preprotésica inferior.
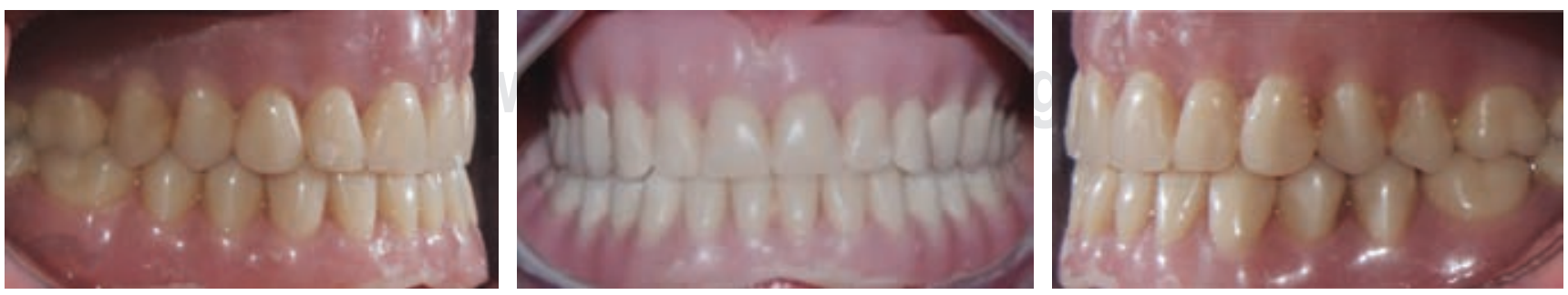

Figura 9: Colocación de prótesis inmediata inferior. 
que ayudará a la elaboración de las prótesis inmediatas (Figura 4).

\section{Cirugía preprotésica superior y colocación de prótesis inmediata}

Se realizaron las cirugías preprotésicas, se inició con la arcada superior en la cual se realizaron extracciones múltiples, regularización del proceso y frenectomía del frenillo labial. Al finalizar el acto quirúrgico se colocó acondicionador de tejidos a la prótesis inmediata superior con el fin de producir presión en el proceso alveolar, disminuir la inflamación que produciría la cirugía y acondicionar los tejidos para las prótesis definitivas (Figuras 5 a 7 ).

\section{Cirugía preprotésica inferior y colocación de prótesis inmediata}

Una semana después se realizó la cirugía preprotésica en la arcada inferior y se retiraron puntos de la arcada superior. Al finalizar el acto quirúrgico se colocó acondicionador de tejidos a la prótesis inmediata inferior con el fin de producir presión en el proceso alveolar, disminuir la inflamación que produciría la cirugía, acondicionar los tejidos para las prótesis definitivas y establecer la oclusión entre ambas prótesis (Figuras 8 y 9 ).

\section{Elaboración y colocación de prótesis definitivas}

Cuatro meses después se procedió a realizar las prótesis totales definitivas. Se inició valorando los rebordes (Figura 10). Después, se procedió a tomar impresiones anatómicas superior e inferior en las cuales se realizaron los portaimpresiones individuales; la rectificación de bordes se realizó con polivinilsiloxano Variotime Easy Putty ${ }^{\circledR}$ a excepción de la zona de sellado posterior

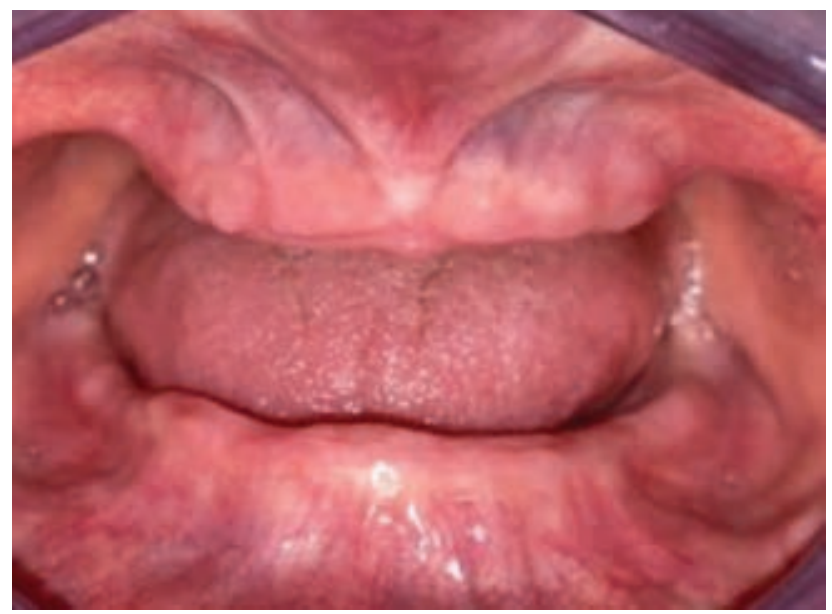

Figura 10: Valoración de los rebordes.

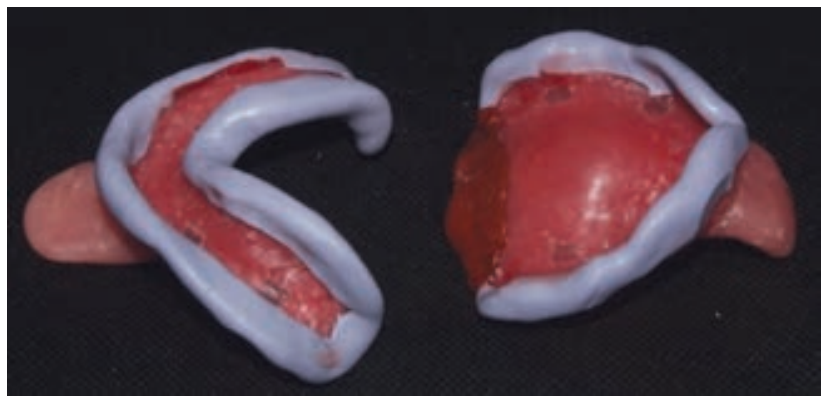

Figura 11: Rectificación de bordes superior e inferior.
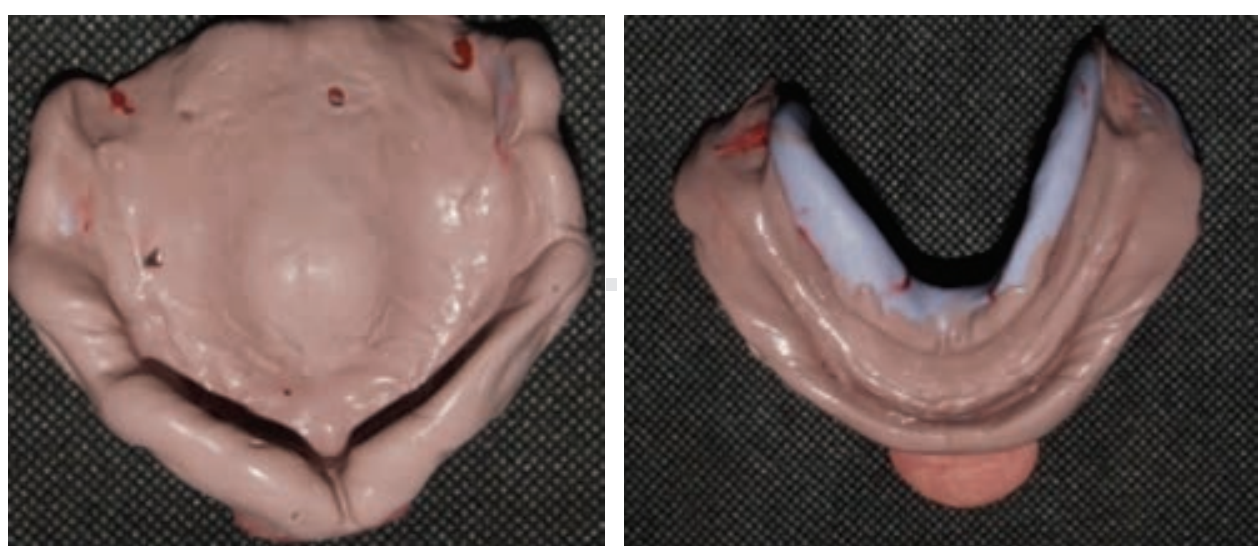

Figura 12:

Toma de impresión fisiológica superior e inferior. 
(postdam) que se hizo con modelina; previamente se colocó VPS Tray Adhesive de $3 \mathrm{M}^{\circledR}$ en los portaimpresiones para evitar el desalojo del material (Figura 11).
Se tomó la impresión fisiológica con Imprint Heavy Body de $3 \mathrm{M}^{\circledR}$, lo cual generó los modelos fisiológicos (Figuras 12 y 13).

Figura 13:

Modelos fisiológicos superior e inferior.
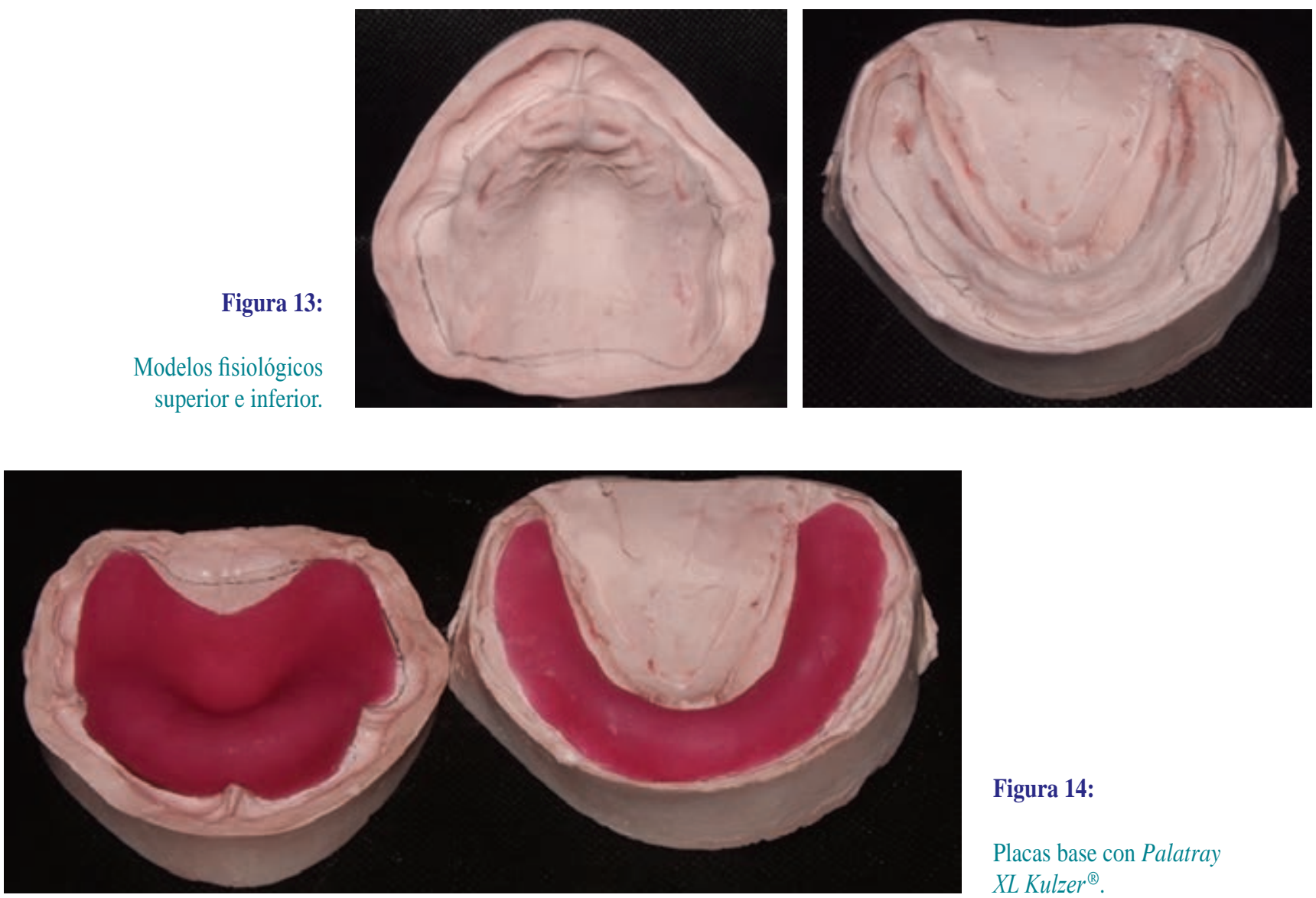

Figura 14:

Placas base con Palatray XL Kulzer ${ }^{\circledR}$.

Figura 15:

Transporte de los rodillos y las relaciones intermaxilares al arco facial.
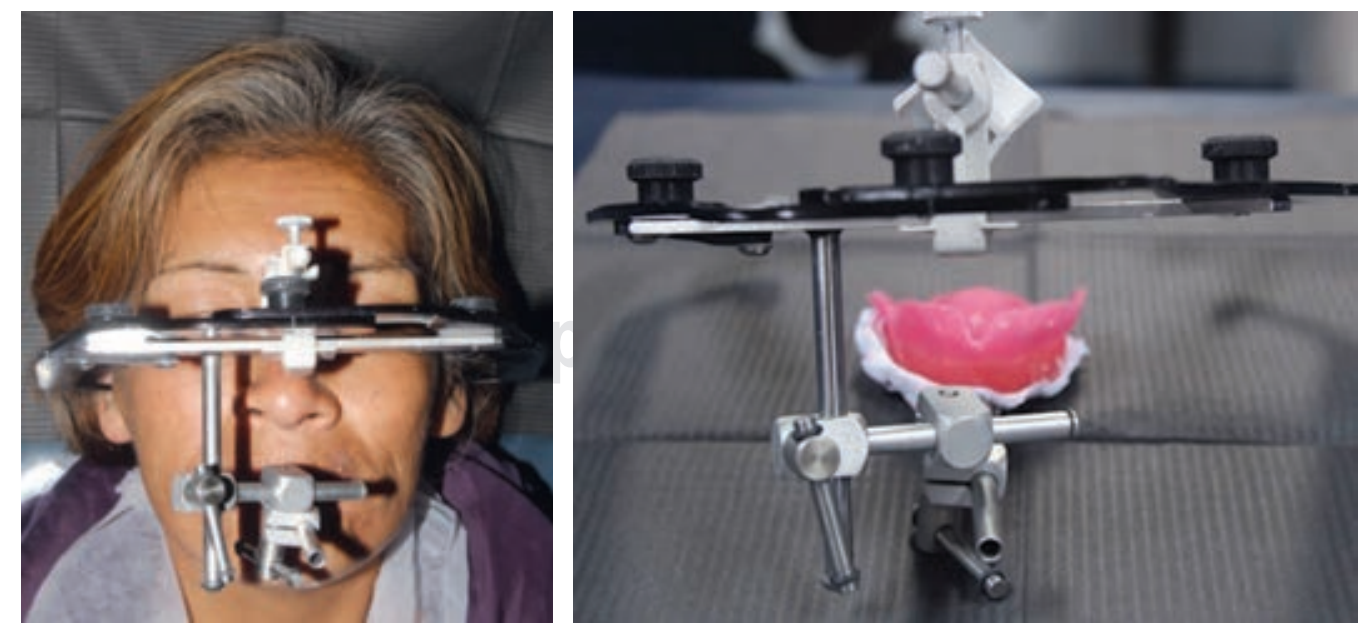
Las placas bases se realizaron con Palatray XL de Kulzer $^{\circledR}$ (Figura 14), en las cuales se colocaron los rodillos y se procedió a la orientación de éstos para tomar las relaciones intermaxilares fisiológicas y se transportaron al arco facial (Figura 15) para el posterior montaje de los dientes en el articulador Whip Mix $8500^{\circledR}$ (Figura 16) y la prueba de dientes en cera (Figura 17).

En el procesado de las prótesis se realizó un caracterizado intrínseco con Opti-Cryl (Figura 18) y por último se colocaron las prótesis y se rectificó la oclusión biba- lanceada en movimientos excéntricos (Figura 19). Los controles de ambas prótesis totales se hicieron tres días después de la colocación, a la primera y tercera semana, y luego cada tres meses.

\section{Fotos comparativas}

En las siguientes imágenes se muestran las fotos intraorales y extraorales comparativas en la transición del tratamiento integral (Figuras 20 y 21).
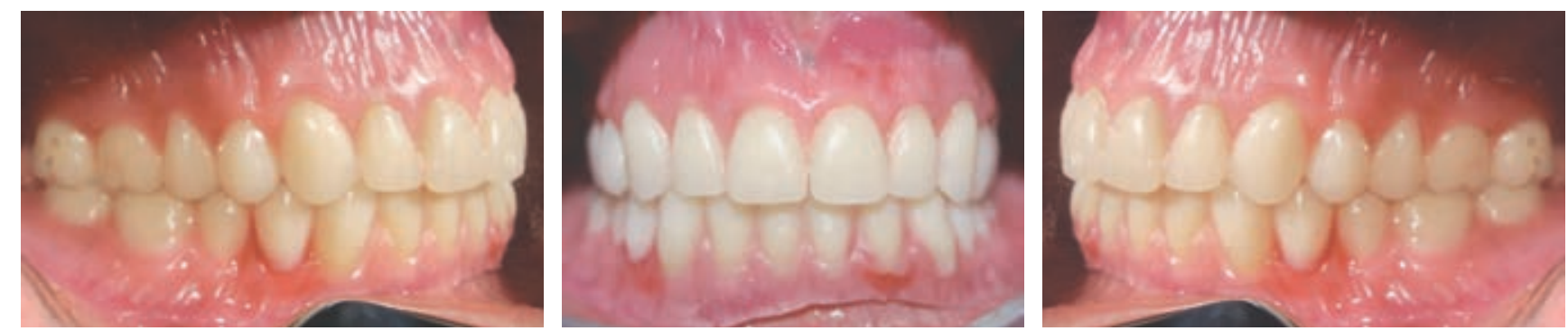

Figura 17: Prueba de dientes en cera en el paciente.
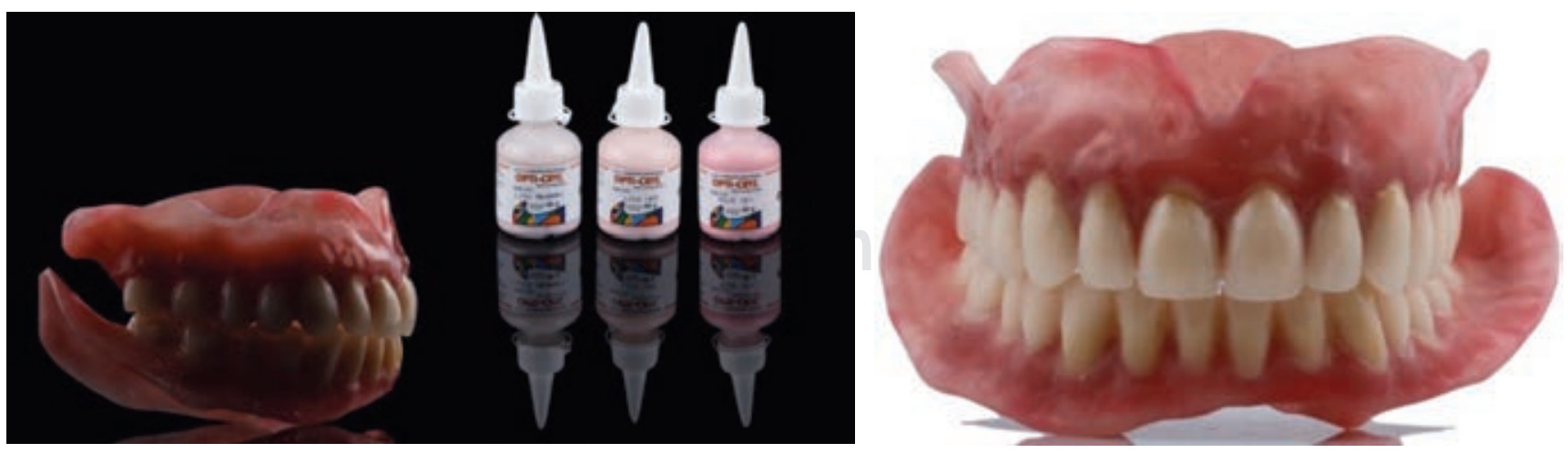

Figura 18: Caracterización intrínseca en prótesis definitivas. 

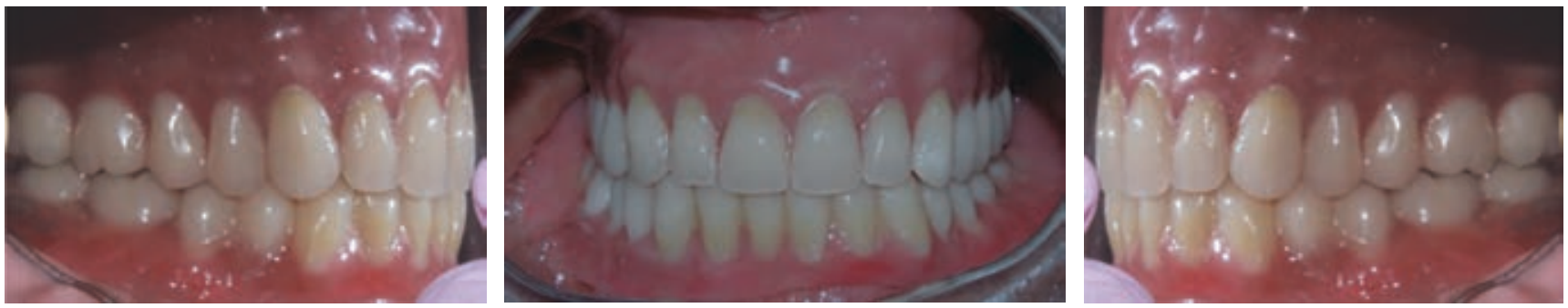

Figura 19: Colocación de prótesis totales definitivas.

Figura 20:

Fotos intraorales comparativas.
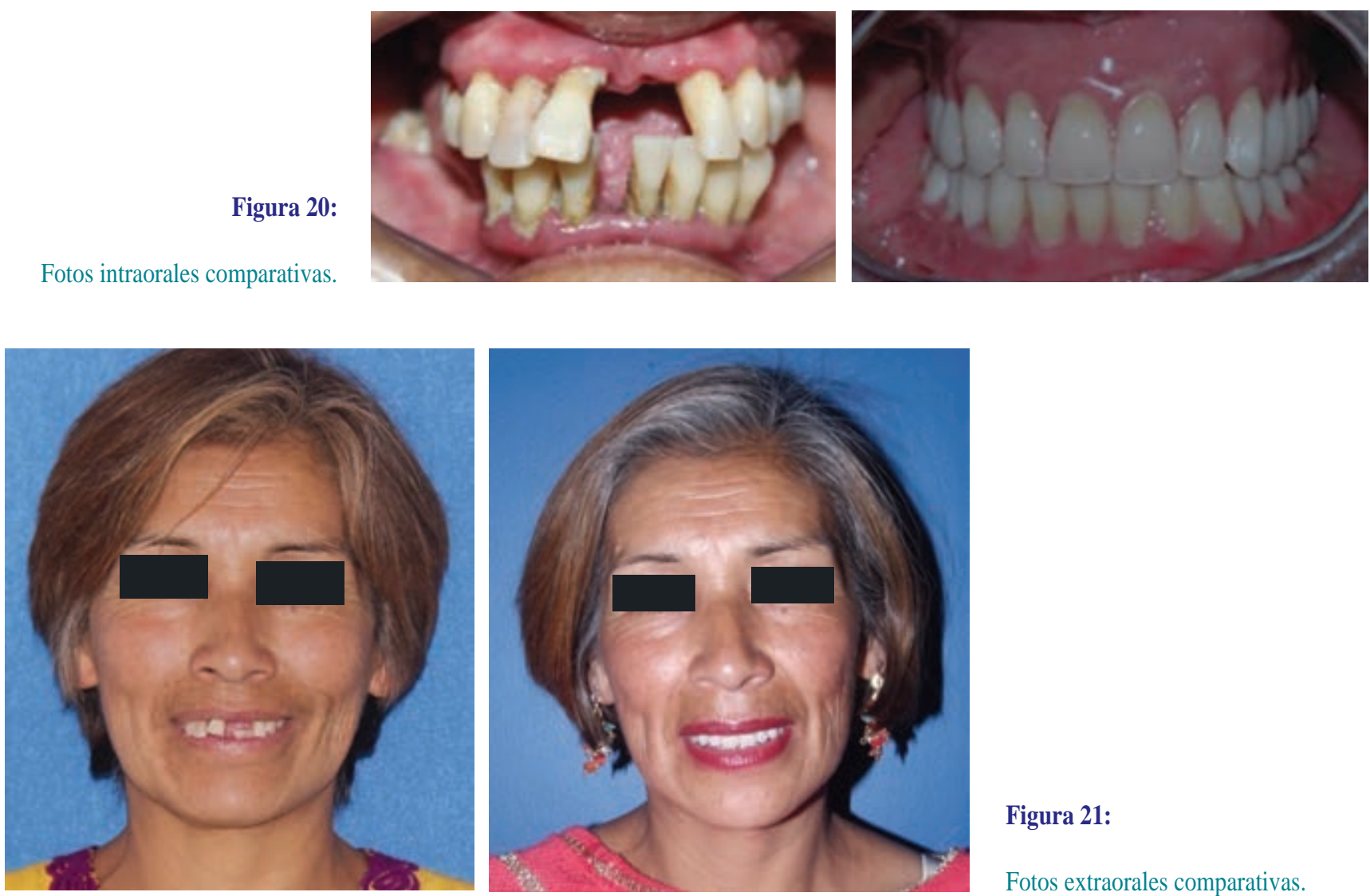

Figura 21:

Fotos extraorales comparativas.

\section{DISCUSIÓN}

La satisfacción del paciente suele ser el pilar fundamental para el éxito del tratamiento. Los pacientes con dentaduras completas suelen tener una mejor aceptación y percepción de las prótesis que los mismos dentistas o técnicos dentales, ${ }^{13}$ la fonética mejora de manera enorme con la adaptación progresiva de las prótesis totales, además de mejorar la alimentación, la nutrición y, por ende, la dieta del paciente. ${ }^{14,15}$
En la actualidad, los clínicos se enfrentan a problemas a la hora de decidir el tratamiento adecuado que se adapte a las necesidades de cada paciente. La rehabilitación de edéntulos totales con implantes dentales son una alternativa predecible desde el primer estudio reportado por Branemark en 1977, ya que proporcionó al paciente estabilidad y retención de ambas prótesis; de este modo se restablece la función masticatoria del desdentado total, se recupera confort y estética, mejora su calidad de vida e incrementa la autoestima y 
seguridad de los pacientes. ${ }^{16}$ Sin embargo, las prótesis totales convencionales siguen siendo una alternativa de tratamiento viable, al seguir el protocolo de diseño protésico determinado con parámetros de calidad técnica, nos conduce a mejorar la calidad de vida con un óptimo nivel de salud oral. ${ }^{17}$

Por último, las prótesis totales deben ser revisadas periódicamente además de controlar la salud de los tejidos mucosos, así como la retención, estabilidad y oclusión de las prótesis totales; los problemas postoperatorios clásicos como las fracturas de las bases protésicas y las pérdidas de los dientes artificiales no están exentos de poder suceder. ${ }^{18,19}$

\section{CONCLUSIONES}

La valoración, diagnóstico y planificación del caso clínico debe ser realizada por un equipo interdisciplinario con el fin de lograr un resultado integral favorable. Al aplicar los conocimientos científicos, teóricos y prácticos se consiguen tratamientos con mayor éxito, devolviendo la funcionalidad masticatoria, fonética y estética.

De la misma manera, hubo un cambio favorable en el factor psicosocial del paciente con los alcances obtenidos en su rehabilitación.

\section{REFERENCIAS}

1. Sánchez ZYE, Ozawa MJL. Estrategias interdisciplinarias para el éxito en la rehabilitación bucal de un paciente adulto joven - parcialmente edéntulo. Reporte de un caso. Implantol Act. 2012; 7 (12): 28-37.

2. Castro RY, Paz FJJ. Secuencia clínica de la rehabilitación del edéntulo total bimaxilar. Reporte de caso clínico. Acta Odontológica Venezolana. 2013; 51 (3). Disponible en: https:// www.actaodontologica.com/ediciones/2013/3/art-15/.

3. Koyano K, Tsukiyama Y, Kuwatsuru R. Rehabilitation of occlusionscience or art? J Oral Rehabil. 2012; 39 (7): 513-521.

4. Utrera LMN, Ozawa MJL. Tratamiento interdisciplinario prótesis bucal-periodoncia de paciente con periodontitis agresiva. Rev Odontol Mex. 2011; 15 (1): 53-61.

5. GBD 2017 Disease and Injury Incidence and Prevalence Collaborators. Global, regional, and national incidence, prevalence, and years lived with disability for 354 diseases and injuries for 195 countries and territories, 1990-2017: a systematic analysis for the Global Burden of Disease Study 2017. Lancet. 2018; 392: 1789-8583.

6. Montaño TVA, Armas VAC, Dourado LA, Salazar GMT. Restablecimiento oclusal mediante procedimientos multidisciplinarios. Rev Cubana Estomatol. 2020: 57 (1).
7. Fuertes RL, Del Valle ZO, Justo DM. Rehabilitación con prótesis inmediata en pacientes geriátricos: (presentación de un caso). Revista Habanera de Ciencias Médicas. 2007: 6 (2).

8. Limonta BL, Dávila FMC, Castillo CL, Pérez BBR, Rodríguez MG. Ventajas de la prótesis inmediata. MEDISAN. 2006: 10.

9. De Lucena SC, Gomes SG, Da Silva WJ, Del Bel Cury AA. Patient's satisfaction and functional assessment of existing complete dentures: correlation with objective masticatory function. J Oral Rehabil. 2011; 38 (6): 440-446.

10. Paleari AG, Marra J, Rodríguez LS, De Souza RF, Pero AC, Mollo FA, Compagnoni MA. A cross-over randomized Clinical trial of eccentric occlusion in complete dentures. J Oral Rehabil. 2012; 39 (8): 615-622.

11. Fradeani M. Análisis estético: un acercamiento al tratamiento protésico. Volumen I. Barcelona, España. Editorial: Quintessence. 2006.

12. Tonetti MS, Greenwell H, Kornman KS. Periodontitis case definition: Framework for staging and grading the individual periodontitis case. J Periodontol. 2018; 45: S149-S161.

13. Marachlioglou CR, Dos Santos JF, Cunha VP. Expectations and final evaluation of complete dentures by patients, dentist and dental technician. J Oral Rehabil. 2010; 37 (7): 518-524.

14. Stelzle F, Ugrinovic B, Knipfer C, Bocklet T, Noth E, Schuster M et al. Automatic, computer-based speech assessment on edentulous patients with and without complete dentures-preliminary results. J Oral Rehabil. 2010; 37 (3): 209-216.

15. Tsakos G, Herrick K, Sheiham A, Watt R: Edentulism and fruit and vegetable intake in low-income adults. J Dent Res. 2010; 89 (5): 462-467.

16. Velasco E, Pato J, García A, Lorrio JM, Cruz JM. El tratamiento con implantes en pacientes edéntulos totales mandibulares mediante rehabilitación fija. Av Periodon Implantol. 2007; 19 (3): 151-159.

17. Medrano NN, Ruiz CM, Gutiérrez PF. Impacto en la calidad de vida relacionada con salud bucal de la rehabilitación protésica convencional en pacientes desdentados completos, atendidos en el servicio público chileno. Revista Científica Mundo de la Investigación y el Conocimiento. 2017; 1 (4): 960-982.

18. Dorner S, Zeman F, Koller M, Lang R, Handel G, Behr M. Clinical performance of complete dentures: a retrospective study. Int J Prosthodont. 2010; 23 (5): 410-417.

19. Avinash SB, Thomas DT, John RA. Computer-aided technology for fabricating complete dentures: Systematic review of historical background, current status, and future perspectives. J Prosthet Dent. 2013; 109 (6): 361-366.

Correspondencia:

Mónica Badillo Barba

E-mail: babm_1985@hotmail.com

Conflicto de intereses: Los autores declaran no tener ningún conflicto de intereses.

Aspectos éticos: Ninguno.

Financiamiento: Ninguno. 